\title{
ON AVERAGING LEFSCHETZ NUMBERS
}

\author{
U. KURT SCHOLZ ${ }^{1}$
}

\begin{abstract}
Let $(E, p, X)$ be a regular covering space where $E$ is a connected metric ANR (absolute neighborhood retract) and let $f: X \rightarrow X$ be a map. This paper investigates the relationship between the Lefschetz number of $f$ and those of its lifts, i.e. maps $f^{\prime}: E \rightarrow E$ so that $p f^{\prime}=f p$. In particular, it is shown that to a lift $f^{\prime}: E \rightarrow E$ one may associate a class of lifts $\mathfrak{L}\left(f^{\prime}\right)$ with the property that the Lefschetz number of $f$ is equal to the average of the Lefschetz numbers of maps in $\mathscr{L}\left(f^{\prime}\right)$.
\end{abstract}

1. Introduction and preliminaries. If $E$ is a compact connected regular covering space of a finite simplicial complex $K$ and $f: K \rightarrow K$ is a map, then the Lefschetz number of $f$ is equal to the average of those of all its lifts (Jiang [8], McCord [9]). In this paper we prove a stronger version of this result, removing the compactness condition and generalizing to regular covers which are connected metric ANR's. Of course in this case, one cannot consider the average of all the lifts, since there will be infinitely many of them.

For a covering space $(E, p, X)$, we shall always assume that $E$ (and hence $X$ ) is a pathwise connected space so that once a basepoint $x_{0} \in X$ has been chosen, the fundamental group of $X$ can be written simply as $\pi(X)$. Similarly, we choose a fixed $e_{0} \in p^{-1}\left(x_{0}\right)$ and use the same convention on $E$. Then each $\& \in \pi(X)$ can be regarded as a covering transformation on $E$, acting transitively on the fibers.

If $(E, p, X)$ is a regular covering space and $f: X \rightarrow X$ is a map, we assume that the base point $x_{0} \in X$ has been chosen to be a fixed point of $f$ (if one exists). We let $C(f, X)$ denote the index of the group $f_{\#}(\pi(X)) \cap p_{\#}(\pi(E))$ in $f_{\#}(\pi(X))$. We also use the notation $\left[f_{\#}(\pi(X)): f_{\#}(\pi(X)) \cap p_{\#}(\pi(E))\right]$ for the index.

Let $f^{\prime}: E \rightarrow E$ be a lift of $f$; then for each $\alpha \in \pi(X)$, the map $f^{\prime} \circ \alpha: E \rightarrow E$

Presented to the Society, January 18, 1972 under the title Averaging Lefschetz numbers of maps; received by the editors July 23, 1971.

AMS 1970 subject classifications. Primary 55H25, 55F05; Secondary 54C55.

Key words and phrases. Lefschetz number, regular covering space, fixed point index.

1 This work was supported by NSF grant \#GU1534. The author wishes to thank Professor Robert F. Brown for helpful suggestions relating to this paper.

(c) American Mathematical Society 1972 
will also be a lift having the same image as $f^{\prime}$ on each fiber. Let $\mathfrak{L}\left(f^{\prime}\right)=$ $\left\{f^{\prime} \alpha: \alpha \in \pi(X)\right\}$.

Suppose now that $X$ is a metric ANR and $f: X \rightarrow X$ is a compact map (i.e. $\operatorname{cl}(f(X))$ is compact). Let $f_{*, p}: H_{p}(X) \rightarrow H_{p}(X)$ denote the induced homomorphism on the singular rational homology groups of $X$. Then $\left\{f_{*, p}\left(H_{p}(X)\right)\right\}$ is of finite type, i.e. $f_{*, p}\left(H_{p}(X)\right)=0$ for all but a finite number of integers $p$ and $f_{*, p}\left(H_{p}(X)\right)$ is finitely generated for all $p$. Thus $f_{*, p}$ has a well defined trace namely that of $f_{*, p} \mid f_{*}\left(H_{p}(X)\right): f_{*}\left(H_{p}(X)\right) \rightarrow f_{*, p}\left(H_{p}(X)\right)$. The nonvanishing of the Lefschetz number $L(f)=\sum(-1)^{p} \operatorname{trace}\left(f_{*, p}\right)$ is a sufficient condition for the existence of fixed points of the map $f: X \rightarrow X$ (Granas [3]).

We note that if $E$ is an ANR and $E$ is a covering space of $X$ then $X$ is also an ANR since it is locally an ANR (Hanner [4]).

2. The main theorem. The main result of this paper is the following:

THEOREM 1. Let $X$ be a compact metric $A N R$ and let $f: X \rightarrow X$ be a map. If $E$ is a connected $A N R$ and $E$ is a regular covering space over $X$ such that $C(f, X)<\infty$, then for any lift $f^{\prime}: E \rightarrow E$ of $f$, each of the following conditions hold:

(1) $f^{\prime}$ is a compact map;

(2) the cardinality of $\mathfrak{L}\left(f^{\prime}\right)$ (written $\left.\left|\mathfrak{L}\left(f^{\prime}\right)\right|\right)$ is equal to $C(f, X)$;

(3) $L(f)$ is equal to the average of the Lefschetz numbers of the maps in $\mathfrak{L}\left(f^{\prime}\right)$.

We first prove parts (1) and (2) of Theorem 1.

Lemma 1. Let $f: X \rightarrow X$ be a map on a compact space and let $E$ be a regular connecte'd cover of $X$ such that $C(f, X)<\infty$. Then any lift $f^{\prime}: E \rightarrow E$ is a compact map and $C(f, X)=\left|\mathcal{L}\left(f^{\prime}\right)\right|$.

Proof. For a given lift $f^{\prime}: E \rightarrow E$, we first show that the set $f^{\prime}\left(p^{-1}(x)\right)$ is finite for any $x \in X$. Let $e \in p^{-1}(x)$ and for each $y \in p^{-1}(x)$ let $\sigma_{y}$ be a path from $e$ to $y$. If $p: E \rightarrow X$ is the covering map, then $p \sigma_{y}$ is a loop based at $x$. The homotopy classes of loops $\left\{\left[p \sigma_{y}\right] \mid y \in p^{-1}(x)\right\}$ has the same cardinality as $p^{-1}(x)$, namely $\left[\pi(X): p_{\#}(\pi(E))\right]$. Consequently the collection $\left\{\left[f p \sigma_{y}\right] \mid y \in p^{-1}(x)\right\} \bmod p_{\#}(\pi(E))$, has cardinality $\left[f_{\#}(\pi(X)): f_{\#}(\pi(X)) \cap\right.$ $\left.p_{\#}(\pi(E))\right]=C(f, X)<\infty$; but this is the cardinality of $f^{\prime}\left(p^{-1}(x)\right)$.

Now for each $x \in f(X)$, let $U_{x}$ be an open connected neighborhood of $x$, evenly covered by $p$. Select an open cover $\mathscr{V}_{x}=\left\{V_{x}^{j}\right\}$ of $f^{-1}(x)$ so that each $V_{x}^{j} \in \mathscr{V}_{x}$ is connected, evenly covered by $p$ and $\operatorname{cl}\left(f\left(V_{x}^{j}\right)\right) \subseteq U_{x}$. Since $X$ and $f(X)$ are compact, we can choose $x_{1}, \cdots, x_{r} \in f(X)$ so that $U_{x_{1}}, \cdots, U_{x_{n}}$ covers $f(X)$ and so that a finite collection of $V_{x_{i}}^{j}$, say $\left\{V_{x_{1}}^{1}, \cdots, V_{x_{1}}^{n_{1}}, V_{x_{2}}^{1}\right.$, $\left.\cdots, V_{x_{r}}^{n_{r}}\right\}$ covers $X$. Write $U_{x_{i}}=U_{i}$ and $V_{x_{i}}^{j}=V_{i j}$. Now if $V_{i j}^{\prime}$ is a component 
of $p^{-1}\left(V_{i j}\right)$, we easily see that $\operatorname{cl}\left(f^{\prime}\left(V_{i j}^{\prime}\right)\right)$ is contained in some component of $p^{-1}\left(U_{i}\right)$. It then follows easily from the first assertion of this proof that $\operatorname{cl}\left(f^{\prime}\left(p^{-1}\left(V_{i j}\right)\right)\right)$ is contained in a finite number (namely $\left.C(f, X)\right)$ of components of $p^{-1}\left(U_{i}\right)$. Thus $\mathrm{cl}\left(f^{\prime}\left(p^{-1}\left(V_{i j}\right)\right)\right)$ is compact. Finally, taking the finite union of these sets, we conclude that $\operatorname{cl}\left(f^{\prime}(E)\right)$ is compact.

The assertion that $\left|\mathfrak{L}\left(f^{\prime}\right)\right|=C(f, X)$ is obvious since $\left|\mathfrak{L}\left(f^{\prime}\right)\right|=$ $\left|f^{\prime}\left(p^{-1}(x)\right)\right|=C(f, X)$.

The basic tool required to prove our main result is the fixed point index for cornpact maps. Let $f: X \rightarrow X$ be a map and let $U$ be an open subset of $X$. The triple $(X, f, U)$ is called admissible if $X$ is a metric ANR and $f$ is a compact map which has no fixed points on the boundary of $U$. Let $C$ be the collection of all admissible triples.

TheOREM 2 (Granas [3]). There is a function $i: C \rightarrow$ integers, satisfying the following five properties:

(1) (Excision) If $(X, f, U)$ and $(X, g, U)$ are in $C$ and $f=g$ on $\mathrm{cl}(U)$, then $i(X, f, U)=i(X, g, U)$.

(2) (Additivity) If $(X, f, U) \in C$ and $U_{1}, \cdots, U_{n}$ are mutually disjoint open subsets of $U$ such that $f$ is fixed point free on $U-\cup_{j=1}^{n} U_{j}$ then

$$
i(X, f, U)=\sum_{j=1}^{n} i\left(X, f, U_{j}\right) .
$$

(3) (Homotopy) If $H: X \times I \rightarrow X$ is a compact map and for each $t \in I$, $\cdot\left(X, h_{t}, U\right) \in C$ (where $\left.h_{t}(x)=H(x, t)\right)$ then $i\left(X, h_{0}, U\right)=i\left(X, h_{1}, U\right)$.

(4) (Normalization) $i(X, f, X)=L(f)$.

(5) (Commutativity) If $f: X \rightarrow Y$ and $g: Y \rightarrow X$ are maps such that $(X, g f, U)$ and $\left(Y, f g, g^{-1}(U)\right)$ are in $C$ then $i(X, g f, U)=i\left(Y, f g, g^{-1}(U)\right)$.

We can now prove Theorem 1 for the special case when $X$ is a connected polyhedron.

Theorem 3 (COMPARE Jiang [8, Theorem 5.2]; MCCord [9]). Let $f: K \rightarrow K$ be a map on a compact polyhedron. If $E$ is a connected regular covering space over $K$ such that $C(f, K)<\infty$, then for any lift $f^{\prime}: E \rightarrow E$ the conclusions of Theorem 1 are valid.

Proof. By moving $f$ through a homotopy, we may assume that $f$ has only a finite number of fixed points, say $x_{1}, \cdots, x_{n}$ occurring in (open) maximal simplices $\sigma_{1}, \cdots, \sigma_{n}$ (Hopf [6]). Select open sets $U_{1}, \cdots, U_{n}$ evenly covered by $p$ so that $x_{j} \in U_{j} \subseteq \sigma_{j}$ for $j=1, \cdots, n$. Now for each $e \in f^{\prime}\left(p^{-1}\left(x_{j}\right)\right)$, there is precisely one map $f_{i}^{\prime} \in \mathscr{L}\left(f^{\prime}\right)$ which has $e$ as a fixed point. Let $U_{j}^{\prime}$ be the component of $p^{-1}\left(U_{j}\right)$ containing $e$. Using the commutative property of the fixed point index, it is not difficult to show 
that $i\left(E, f_{i}^{\prime}, U_{j}^{\prime}\right)=i(K, f, U)$. Since $f^{\prime}\left(p^{-1}(x)\right)$ contains exactly $C(f, X)=$ $\left|\mathfrak{L}\left(f^{\prime}\right)\right|$ points (Lemma 1$)$, we have

$$
i\left(K, f, U_{j}\right)=\sum_{f^{\prime} \in \mathbb{E}\left(f^{\prime}\right)} \frac{i\left(E, f_{i}^{\prime}, p^{-1}\left(U_{j}\right)\right)}{\left|\mathfrak{L}\left(f^{\prime}\right)\right|} .
$$

Taking the sum over all $U_{j}$ we obtain

$$
\begin{aligned}
L(f)=\sum_{j=1}^{n} i\left(K, f, U_{j}\right) & =\sum_{j=1}^{n}\left(\sum_{f^{\prime} \in \dot{\mathcal{L}}^{\prime}\left(f^{\prime}\right)} \frac{i\left(E, f_{i}^{\prime}, p^{-1}\left(U_{j}\right)\right)}{\left|\mathfrak{Q}\left(f^{\prime}\right)\right|}\right) \\
& =\sum_{f i^{\prime} \in \mathcal{Q}^{\prime}\left(f^{\prime}\right)} \frac{L\left(f_{i}^{\prime}\right)}{\left|\mathcal{Q}\left(f^{\prime}\right)\right|} \cdot \square
\end{aligned}
$$

REMARK. It has been tacitly assumed that $E$ is a metrizable ANR. This is indeed the case since $E$ is imbeddable in $R^{m}$ for some $m$ (e.g. $m=1+$ $2 \operatorname{dim} K)$ and any locally finite polyhedron is an ANR [4].

ProOf OF THEOREM 1 COMPLETED. Let $E$ be a regular covering space and $f: X \rightarrow X$ a map so that $C(f, X)<\infty$. Using the domination theorem for ANR's [4], there exists a compact polyhedron $K$ and maps $\phi: X \rightarrow K$, $\psi: K \rightarrow X$ so that $\psi \circ \phi: X \rightarrow X$ is homotopic to the identity map on $X$. Since $X$ is connected we may assume $K$ is also (by restricting our attention to a component of $K$ containing $\phi(X))$. Note that $\psi_{\#}^{\circ} \phi_{\#}$ is equal to the identity on $\pi(X)$.

Let $N$ be the kernel of the map

$$
\pi(K) \stackrel{\psi_{\#}}{\longrightarrow} \pi(X) \stackrel{\text { projection }}{\longrightarrow} \frac{\pi(X)}{p_{\#}(\pi(E))} .
$$

Clearly $\phi_{\#}^{\circ} p_{\#}(\pi(E)) \subseteq N$ so that applying $\psi_{\#}$ to each side gives $p_{\#}(\pi(E)) \subseteq$ $\psi_{\#}(N)$. On the other hand, $\psi_{\#}(N) \subseteq p_{\#}(\pi(E))$ by definition. Thus $\psi_{\#}(N)=$ $p_{\#}(\pi(E))$.

Proceeding with the proof of Theorem 1, we construct a connected regular cover $\left(E_{K}, p, K\right)$ of $K$ so that $p_{\#} \tau\left(\left(E_{K}\right)\right)=N$. Since $\phi_{\#} p_{\#}(\pi(E)) \subseteq$ $p_{\#} \pi\left(E_{K}\right)$ and $\psi_{\#}\left(p_{\#} \pi\left(E_{K}\right)\right)=p_{\#}(\pi(E))$ we can apply the lifting theorem for covering spaces $\left[5\right.$, p. 256] to obtain maps $\phi^{\prime}: E \rightarrow E_{K}$ and $\psi^{\prime}: E_{K} \rightarrow E$ making the following diagram commute:

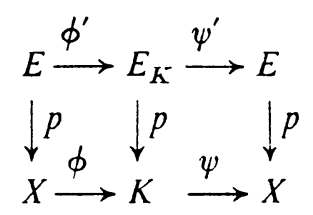


Since $\psi \circ \phi$ is homotopic to the identity map on $X$, it follows that $\psi^{\prime} \circ \phi^{\prime}$ is homotopic to some covering transformation. We may, in fact, assume that $\psi^{\prime} \circ \phi^{\prime}$ is actually homotopic to the identity map on $E$ (otherwise, replace $\psi^{\prime}$ by $\alpha \circ \psi^{\prime}$ for some $\left.\alpha \in \pi(x)\right)$.

Now, given $f: X \rightarrow X$ so that $C(f, X)<\infty$, define $\bar{f}: K \rightarrow K$ by $\bar{f}=\phi \circ f \circ \psi$. We claim that $C(f, X)=C\left(\bar{f}, K^{\prime}\right)$. This fact is immediate by observing that $\phi_{\#}$ induces an isomorphism:

$$
\frac{f_{\#}(\pi(X))}{f_{\#}(\pi(X)) \cap p_{\#}(\pi(E))} \rightarrow \frac{\bar{f}_{\#}(\pi(K))}{f_{\#}(\pi(K)) \cap p_{\#}\left(\pi\left(E_{K}\right)\right)} .
$$

It is clear that $\mathfrak{L}\left(\phi^{\prime} f^{\prime} \psi^{\prime}\right)=\left\{\phi^{\prime} f_{i}^{\prime} \psi^{\prime}: f_{i}^{\prime} \in \mathfrak{L}\left(f^{\prime}\right)\right\}$ and hence by Theorem 3 , we deduce:

$$
L(\bar{f})=\sum \frac{L\left(\phi^{\prime} f_{i}^{\prime} \psi^{\prime}\right)}{\left|\mathfrak{L}\left(\phi^{\prime} f^{\prime} \psi^{\prime}\right)\right|} .
$$

Since $\psi_{*, p}^{\circ} \phi_{*, p}$ and $\psi_{*, p}^{\prime} \phi_{*, p}^{\prime}$ are the identity isomorphisms, it follows readily that

$\operatorname{trace}\left(\bar{f}_{*, p}\right)=\operatorname{trace}\left(f_{*, p}\right)$ and $\operatorname{trace}\left(\phi_{*, p}^{\prime} \cdot\left(f_{2}^{\prime}\right)_{*, p} \cdot \psi_{*, p}^{\prime}\right)=\operatorname{trace}\left(\left(f_{i}^{\prime}\right)_{*, p}\right)$.

Fitting everything together and using the fact that $\left|\mathfrak{L}\left(\phi^{\prime} f^{\prime} \psi^{\prime}\right)\right|=\left|\mathfrak{L}\left(f^{\prime}\right)\right|$ we obtain

$$
L(f)=L(\bar{f})=\sum \frac{L\left(\phi^{\prime} f_{i}^{\prime} \psi^{\prime}\right)}{\left|\mathfrak{L}\left(\phi^{\prime} f^{\prime} \psi^{\prime}\right)\right|}=\sum \frac{L\left(f_{i}^{\prime}\right)}{\left|\mathfrak{L}\left(f^{\prime}\right)\right|} .
$$

4. An example. The following example shows that we can use Theorem 1 to actually compute $L(f)$ in certain cases.

Let $S^{2 n-1}$ be the $2 n-1$ sphere represented by complex coordinates $z_{1}, \cdots, z_{n}$ such that $\sum z_{i} \bar{z}_{i}=1$. Given a positive integer $p$ and integers $q_{1}, \cdots, q_{n-1}$ relatively prime to $p$, define $h: S^{2 n-1} \rightarrow S^{2 n-1}$ by $h\left(z_{1}, \cdots, z_{n}\right)=$ $\left(z_{1} \exp \left(2 \pi q_{1} i \mid p\right), \cdots, z_{n} \exp \left(2 \pi q_{n} i / p\right)\right)$. Then $h$ generates a cyclic group of homeomorphisms acting on $S^{2 n-1}$ without fixed points. The quotient space is the generalized lens space $L=L^{p}\left(q_{1}, \cdots, q_{n}\right)$. We let $\left[z_{1}, \cdots, z_{n}\right] \in L$ denote the image of a point $\left(z_{1}, \cdots, z_{n}\right)$ under the natural projection.

Let $\pi_{1}\left(L \times S^{1}\right) \approx Z_{p} \oplus Z$ be generated by $\alpha$ and $\beta$ respectively, and let $f: L \times S^{1} \rightarrow L \times S^{1}$ be any map so that $f_{\#}(\alpha)=k \alpha$ and $f_{\#}(\beta)=0$. We consider the cover $S^{2 n-1} \times R \rightarrow L \times S^{1}$ given by $\left(z_{1}, \cdots, z_{n}, r\right) \rightarrow\left(\left[z_{1}, \cdots, z_{n}\right]\right.$, $\exp (2 \pi r i))$. Then $C(f, X)<\infty$. Using [9, Theorem 4.1] it follows easily that all lifts of $f$ have the same Lefschetz number. Hence by Theorem 1, for any lift $f^{\prime}$, we have $L(f)=L\left(f^{\prime}\right)$. But $L\left(f^{\prime}\right)=1-\operatorname{deg}\left(f^{\prime}\right)=1-\operatorname{deg}(f)$ (where $\operatorname{deg}(f)=$ degree of $f$ ).

REMARK. If $p$ is odd, it can be shown (using cohomology with $Z_{p}$ coefficients) that $\operatorname{deg}(f) \equiv k^{n}(\bmod p)$. 
A more interesting case occurs when $f_{\#}(\beta)=l \beta$. Then using $S^{2 n-1} \times S^{1}$ as a covering space and the fact that all lifts have the same Lefschetz number [8], an easy computation shows that $L(f)=\left(k^{n}-1\right)(1-l) \bmod p$.

\section{REFERENCES}

1. F. Browder, Asymptotic fixed point theorems, Math. Ann. 185 (1970), 38-61.

2. R. F. Brown, The Lefschetz fixed point theorem, Scott, Foresman, Chicago, Ill., 1971.

3. A. Granas, The Hopf-Lefschetz fixed point theorem for noncompact ANR's, Proc. Conference on Infinite Dimensional Topology, Baton Rouge, La., 1967.

4. O. Hanner, Some theorems on absolute neighborhood retracts, Ark. Mat. 1 (1951), 389-408. MR 13, 266.

5. P. J. Hilton and S. Wylie, Homology theory. An introduction to algebraic topology, Cambridge Univ. Press, New York, 1960. MR 22 \#5963.

6. H. Hopf, Über die algebräische Anzahl von Fixpunkten, Math. Z. 29 (1929), 493-524.

7. S.-T. Hu, Homotopy theory, Pure and Appl. Math., vol. 8, Academic Press, New York, 1959. MR 21 \#5186.

8. Jiang Bo-Ju (Chiang Po-chu), Estimation of the Nielsen numbers, Acta Math. Sinica 14 (1964), 304-312 = Chinese Math. 5 (1964), 330-339. MR 30 \#1510.

9. D. McCord, The converse of the Lefschetz fixed point theorem for surfaces and higher dimensional manifolds, Doctoral Dissertation, University of Wisconsin, Madison, Wis., 1970.

10. U. K. Scholz, The Nielsen theory on fixed point classes for Palais maps, Doctoral Dissertation, University of California, Los Angeles, Calif., 1970.

Department of Mathematics, University of Arizona, Tuscon, Arizona 85721

Current address: Department of Mathematics, Louisiana State University, Baton Rouge, Louisiana 70803 